\title{
Morphological classification of plant cell deaths
}

\author{
WG van Doorn ${ }^{\star, 1}$, EP Beers ${ }^{2}$, JL Dangl $\left.\right|^{3}$, VE Franklin-Tong ${ }^{4}$, P Gallois ${ }^{5}$, I Hara-Nishimura ${ }^{6}$, AM Jones ${ }^{7}$, M Kawai-Yamada $^{8}$, E Lam $^{9}$, \\ $\mathrm{J} \mathrm{Mundy}^{10}$, LAJ Mur ${ }^{11}$, M Petersen $^{10}$, A Smertenko ${ }^{12}$, M Taliansky ${ }^{13}$, F Van Breusegem ${ }^{14}$, T Wolpert $^{15}$, E Woltering ${ }^{16}$, B Zhivotovsky ${ }^{17}$ \\ and PV Bozhkov*,18
}

\begin{abstract}
Programmed cell death (PCD) is an integral part of plant development and of responses to abiotic stress or pathogens. Although the morphology of plant PCD is, in some cases, well characterised and molecular mechanisms controlling plant PCD are beginning to emerge, there is still confusion about the classification of PCD in plants. Here we suggest a classification based on morphological criteria. According to this classification, the use of the term 'apoptosis' is not justified in plants, but at least two classes of PCD can be distinguished: vacuolar cell death and necrosis. During vacuolar cell death, the cell contents are removed by a combination of autophagy-like process and release of hydrolases from collapsed lytic vacuoles. Necrosis is characterised by early rupture of the plasma membrane, shrinkage of the protoplast and absence of vacuolar cell death features. Vacuolar cell death is common during tissue and organ formation and elimination, whereas necrosis is typically found under abiotic stress. Some examples of plant PCD cannot be ascribed to either major class and are therefore classified as separate modalities. These are PCD associated with the hypersensitive response to biotrophic pathogens, which can express features of both necrosis and vacuolar cell death, PCD in starchy cereal endosperm and during self-incompatibility. The present classification is not static, but will be subject to further revision, especially when specific biochemical pathways are better defined.
\end{abstract}

Cell Death and Differentiation (2011) 18, 1241-1246; doi:10.1038/cdd.2011.36; published online 15 April 2011

Research on plant cell death has grown considerably in the past few years, owing to the importance of cell death for plant development and defense. Just as animal cells engage several mechanisms leading to death, the road to cell demise in plants can also vary. The long evolutionary distance and distinct cellular architecture between the two kingdoms may account for the differences between the mechanisms of plant and animal cell death. It is therefore appropriate to assess the relevance of animal cell death nomenclature ${ }^{1}$ to plants. At present, there is confusion in cell death terminology in plant biology, which drives our attempt to formulate a more logical classification. Although our molecular understanding of plant cell death regulation and execution is insufficient to create definitive classifications based on precise biochemical pathways, it is possible to begin classifying plant cell death scenarios based on morphological criteria, as was initially the case in animal cell death research ${ }^{2,3}$ and is still used for the classification of cell death in animal science. ${ }^{1}$

This document attempts to provide a classification of plant cell death. We urge authors, reviewers and editors to follow this classification to facilitate communication between scientists and accelerate research in this field.

\footnotetext{
${ }^{1}$ Mann Laboratory, Department of Plant Sciences, University of California, Davis, CA 95616, USA; ${ }^{2}$ Department of Horticulture, Virginia Polytechnic Institute and State University, Blacksburg, VA 24061, USA; ' ${ }^{3}$ Department of Biology and Carolina Center of Genome Science, University of North Carolina, Chapel Hill, NC 27599, USA; ${ }^{4}$ School of Biosciences, College of Life and Environmental and Life Sciences, University of Birmingham, Edgbaston, Birmingham B15 2TT, UK; ${ }^{5}$ Faculty of Life Sciences, University of Manchester, Manchester M13 9PT, UK; ' ${ }^{6}$ Department of Biological Science, Graduate School of Science, Kyoto University, Sakyo-ku, Kyoto 606-8502, Japan; ${ }^{7}$ Departments of Biology and Pharmacology, University of North Carolina, Chapel Hill, NC 27599, USA; ${ }^{8}$ Department of Environmental Science and Technology, Institute for Environmental Science and Technology, Saitama University, Saitama 338-8570, Japan; ${ }^{9}$ Department of Plant Biology and Pathology, Rutgers the State University of New Jersey, 59 Dudley Road, New Brunswick, NJ 08901, USA; ${ }^{10}$ Department of Biology, University of Copenhagen, Ole Maaloes Vej 5, 220 Copenhagen, Denmark; ${ }^{11}$ Aberystwyth University, Institute of Biological, Environmental and Rural Sciences, Aberystwyth, Wales SY23 3DA, UK; ${ }^{12}$ The Integrative Cell Biology Laboratory, School of Biological and Biomedical Sciences, University of Durham, South Road, Durham DH1 3LE, UK; ${ }^{3}$ Plant Pathology Programme, Scottish Crop Research Institute, Invergowrie, Dundee DD2 5DA, UK; ${ }^{14}$ VIB Department of Plant Systems Biology and Department of Plant Biotechnology and Genetics, University of Ghent, Technologiepark 927, Ghent 9052, Belgium; ${ }^{15}$ Department of Botany and Plant Pathology, Center for Genome Research and Biocomputing, Oregon State University, Corvallis, OR 97330, USA; ${ }^{16}$ Horticultural Supply Chains, Droevendaalsesteeg 1, and Food and Biobased Research, Bornse Weilanden 9 , Wageningen University and Research Center, Wageningen 6708, The Netherlands; ${ }^{17}$ Institute of Environmental Medicine, Division of Toxicology, Karolinska Institutet, Box 210 , Stockholm171 77, Sweden and ${ }^{18}$ Department of Plant Biology and Forest Genetics, Uppsala BioCentre, Swedish University of Agricultural Sciences, Box 7080, Uppsala 75007, Sweden

*Corresponding authors: WG van Doorn, Mann Laboratory, Department of Plant Sciences, University of California, Davis, CA 95616, USA. Tel: +1 461 867 3228; Fax: +1 530752 4554; E-mail: wgvandoorn@ ucdavis.edu or PV Bozhkov, Department of Plant Biology and Forest Genetics, Uppsala BioCentre, Swedish University of Agricultural Sciences, Box 7080, Uppsala 75007, Sweden. Tel: +46 18 673228; Fax: +46 18 673389; E-mail: peter.bozhkov@slu.se

Keywords: apoptosis; autophagy; cell wall; hypersensitive response; necrosis; vacuolar cell death

Abbreviations: GFP, green fluorescent protein; EM, electron microscopy; IF microscopy, immunofluorescent microscopy; HR, hypersensitive response; JC-1, JC-1 mitochondrial membrane potential detection kit based on $5,5^{\prime}, 6,6^{\prime}$-tetrachloro-1,1 $1^{\prime}, 3,3^{\prime}$-tetraethylbenzimidazolylcarbocyanine iodide; MDC, monodansylcadaverine; MMP, mitochondrial membrane permeabilisation; PCD, programmed cell death; RNS, reactive nitrogen species; ROS, reactive oxygen species; SI, self-incompatibility; TMRE, tetramethyl-rhodamine ethyl ester; VPE, vacuolar processing enzyme

Received 09.2.11; accepted 07.3.11; Edited by V De Laurenzi; published online 15.4.11
} 


\section{Absence of Apoptosis in Plants}

Apoptosis is one of the three major types of cell death found in animals. Compared with the other two - autophagic cell death and necrosis - apoptosis is much better understood, both cytologically and biochemically. ${ }^{1,4}$ Apoptosis is accompanied by rounding up of the cell, reduction of cellular volume, chromatin condensation, nuclear segmentation and very little ultrastructural modification of cytoplasmic organelles. Its hallmark is blebbing of the plasma membrane (which maintains its integrity until the final stages of apoptosis), followed by fragmentation of the cell into smaller parcels called apoptotic bodies. Finally, the apoptotic bodies are engulfed by phagocytes and degraded by lysosomal enzymes. This is critical to prevent subsequent induction of inflammation due to leakage of dead cell contents. The term 'apoptosis' should be applied exclusively to cell death that manifests these morphological features. Although apoptosis is often associated with activation of caspases and oligonucleosomal fragmentation of DNA, these processes can also take place during non-apoptotic cell death, and are thus insufficient criteria for assignment. ${ }^{1}$

Plant cells do not exhibit 'classic' apoptosis for the following reasons. First, rigid cell walls preclude the necessity for breakdown of the plant cells into apoptotic bodies. Second, there are no phagocytic cells. A considerable number of articles describing 'plant apoptosis' or 'apoptotic-like programmed cell death (PCD)' have nevertheless been published. Critical analysis of this literature reveals three major points that indicate misuse of the term 'apoptosis'. First, chromatin condensation and DNA fragmentation are often quoted as apoptotic features. However, neither is specific to apoptosis, because they can also be observed during necrosis and autophagic death. ${ }^{5-7}$ Second, stress treatments often induce shrinkage of the plant protoplast, but not of the cell itself, which can be morphologically reminiscent of apoptotic cell shrinkage. However, animal cells that shrink during apoptosis maintain their plasma membrane integrity to form apoptotic bodies, ${ }^{8}$ whereas plant protoplasts that shrink in response to stress usually have damaged plasma membranes and do not fragment further into discrete bodies. ${ }^{9}$ Third, increased caspase-like proteolytic activities (in most cases unlinked to specific proteases) in dying plant cells have been used as an argument for the existence of plant apoptosis. This is an insufficient criterion because activation of caspases per se does not always lead to apoptosis in animal cells. ${ }^{7}$ Furthermore, the activation of plant proteases that possess caspase-like activity has not been shown to lead to apoptotic morphology. ${ }^{10-12}$

\section{Definition of 'Vacuolar' Plant Cell Death}

Plants have elaborate vacuolar systems that, in contrast to animal lysosomes, can occupy most of the plant cell volume. ${ }^{13}$
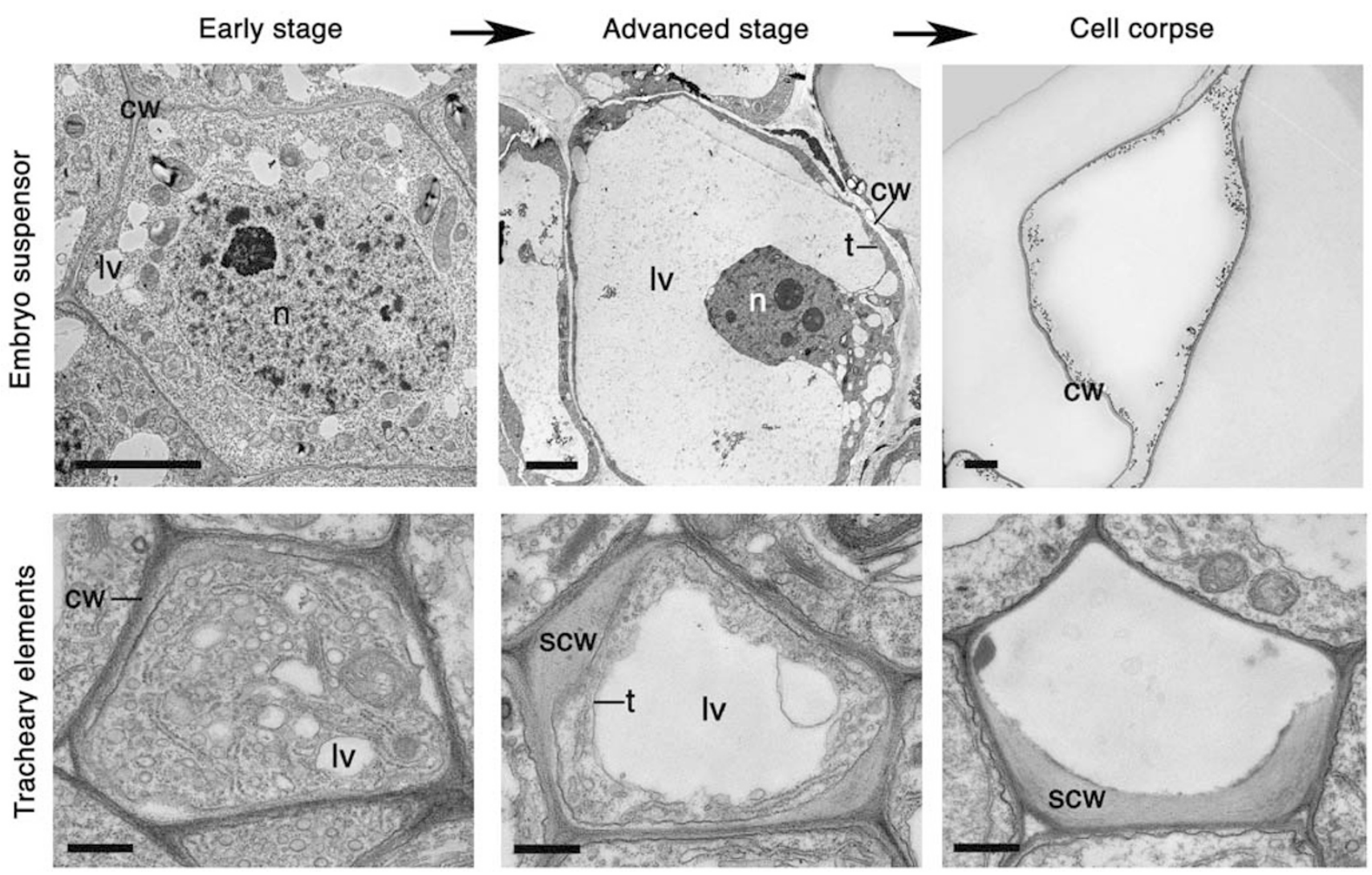

Figure 1 Vacuolar cell death. Electron micrographs of programmed cell death (PCD) in the Norway spruce embryo-suspensor cells ${ }^{17}$ (top panels) and Arabidopsis tracheary elements ${ }^{20}$ (bottom panels). cw, cell wall; Iv, lytic vacuole; $\mathrm{n}$, nucleus; scw, secondary cell wall; t, tonoplast. Scale bars, $5 \mu \mathrm{m}$ (embryo suspensor) and $500 \mathrm{~nm}$ (tracheary elements). Pictures of Norway spruce embryo-suspensor cells were kindly provided by Dr. Lada Filonova and Dr. Elena Minina (Swedish University of Agricultural Sciences, Uppsala, Sweden) and those of Arabidopsis tracheary elements by Dr. Utku Avci (University of Georgia, Complex Carbohydrate Research Center, Athens, GA, USA) 
Similar to the roles of lysosomes in animals, plants also use lytic vacuoles to recycle parts of their cells during normal development and during nutritional stress. ${ }^{14}$ These lytic vacuoles acquire an important function in one major class of plant cell death, which we recommend be termed 'vacuolar cell death'. ${ }^{15}$

Vacuolar cell death is often manifested by a gradual decrease in the volume of the cytoplasm and a concomitant increase in the volume occupied by lytic vacuoles (Figure 1). Engulfment of the cytoplasm by lytic vacuoles with subsequent cargo degradation is a major mechanism of cell dismantling during vacuolar cell death. Electron micrographs often show invaginations in the vacuolar membrane (tonoplast) and fusion of vesicles with the vacuole, followed by uptake and degradation of portions of the cytoplasm in the vacuolar lumen. This process resembles micro- or macroautophagy. ${ }^{16-20}$ The final step in the execution of vacuolar cell death is rupture of the tonoplast, and a massive release of vacuolar hydrolases. These rapidly destroy the entire protoplast or in some cases even the entire cell including the cell wall. Other morphological events during vacuolar cell death include formation of actin cables, nuclear envelope disassembly and, in some examples, nuclear segmentation. The remaining mitochondria and other organelles, as well as the plasma membrane, remain morphologically intact until rupture of the tonoplast (Table 1; Figure 1). A robust approach to diagnose vacuolar cell death would combine electron microscopy (EM) with the analysis of autophagic activity, requirement for vacuolar processing enzymes (VPE) and cytoskeletal changes (Table 2).

Execution of vacuolar cell death may be a slow process that can take several days until the rupture of the tonoplast that accomplishes protoplast clearance. ${ }^{18-21}$ Depending on the system, the cell wall can be largely degraded, as for example during aerenchyma formation, leaf perforations in the lace plant and petal senescence ${ }^{22-24}$ or can remain intact, for example, during xylem differentiation in vascular plants or leaf remodelling in Monstera (Figure 1). ${ }^{24-26}$ Examples of vacuolar cell death are found during embryo, organ and tissue morphogenesis and senescence, and include, in addition to those mentioned above, the formation of embryo-suspensor, pollen, ovary, ducts and laticifers. ${ }^{19}$

Knockout of $A T G$ genes was shown to accelerate Arabidopsis leaf senescence, ${ }^{27,28}$ and ATG5 has recently been found to be required for vacuolar cell death of Arabidopsis tracheary elements. ${ }^{29}$ More extensive work is still needed to determine whether or not ATG-dependent autophagic pathways are required for the execution of vacuolar cell death.

\section{Definition of 'Necrotic' Plant Cell Death}

Necrosis of animal cells is defined morphologically by the lack of apoptotic or autophagic features, and positively by the frequent occurrence of an initial gain in cell volume, swelling of

Table 1 Morphological features of the two major classes of plant cell death

\begin{tabular}{|c|c|c|}
\hline Class of cell death & Features & Notes \\
\hline Vacuolar & $\begin{array}{l}\text { Accumulation of autophagosomes and small lytic vacuoles } \\
\text { Formation of actin cables } \\
\text { Nuclear envelope disassembly } \\
\text { Normal turgor and intact organelles until rupture } \\
\text { of the tonoplast } \\
\text { Formation of large lytic vacuoles } \\
\text { Rupture of tonoplast } \\
\text { Empty-walled cell corpse }\end{array}$ & $\begin{array}{l}\text { The extent of autophagic cell dismantling before the } \\
\text { rupture of tonoplast can vary depending on the system }\end{array}$ \\
\hline Necrosis & $\begin{array}{l}\text { Swelling of mitochondria } \\
\text { Early rupture of plasma membrane } \\
\text { Shrinkage of the protoplast } \\
\text { Cell corpse remains largely unprocessed }\end{array}$ & $\begin{array}{l}\text { This type of cell death is distinguished from accidental or } \\
\text { injury-induced cellular demise due to physical destruction } \\
\text { of cellular integrity. An important feature is the required } \\
\text { participation of cellular components }\end{array}$ \\
\hline
\end{tabular}

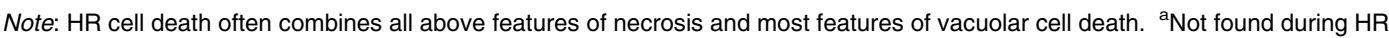

Table 2 Biochemical and cell biological features of the two major classes of plant cell death and their detection methods

\begin{tabular}{|c|c|c|}
\hline Class of cell death & Features & Detection methods \\
\hline \multirow[t]{4}{*}{ Vacuolar } & Autophagic activity & $\begin{array}{l}\text { MDC and lysotracker staining } \\
\text { Atg8-GFP expression } \\
\text { Immunoblotting detection of Atg8 lipidation }\end{array}$ \\
\hline & Acidification of vacuoles & Lysotracker staining \\
\hline & $\begin{array}{l}\text { Reorganisation of } \\
\text { cytoskeleton }\end{array}$ & Staining of F-actin by specific dyes or antibody \\
\hline & Activation of VPE & Colorimetric/fluorogenic substrate-based assays in live cells and cell lysates \\
\hline \multirow[t]{4}{*}{ Necrosis } & MMP & $\begin{array}{l}\text { JC-1 or TMRE staining of mitochondria } \\
\text { IF microscopy or immunoblotting detection of cytochrome } c \text { release }\end{array}$ \\
\hline & Respiratory decline & Oxygen consumption measurement (polarography) \\
\hline & Drop in ATP level & Luminometric assay of intracellular ATP concentration \\
\hline & ROS and RNS accumulation & $\begin{array}{l}\text { In situ detection and spectrofluorometric measurement using } \\
\text { ROS- and/or RNS-sensitive probes }\end{array}$ \\
\hline
\end{tabular}


various organelles, early rupture of the plasma membrane and loss of intracellular content. ${ }^{1,30}$ Although it is no longer considered to be an unprogrammed process, necrosis remains poorly characterised at the biochemical and genetic levels, so there are as yet no molecular markers for it. In animal systems, necrosis is often preceded by an increase in cytosolic calcium ion concentration $\left(\left[\mathrm{Ca}^{2+}\right]_{\text {cyt }}\right)$, lipid degradation and activation of calpain family proteases. Mitochondria and lysosomes have been implicated in the downstream events. Mitochondrial changes include uncoupling of respiration, the production of reactive oxygen species (ROS) and nitrogen species (RNS), a drop in ATP level and mitochondrial membrane permeabilisation (MMP). Lysosomal events include ROS production and permeabilisation of the lysosomal membrane causing release of active cathepsin proteases to the cytosol. ${ }^{1,31}$

Cell death with many of the above characteristics occurs widely in plants. It is induced by a range of abiotic stresses and by successful recognition of a pathogen during the hypersensitive response (HR). It is also found in the cells challenged by necrotrophic pathogens (they are called necrotrophic because they kill host cells to derive nutrients). However, in the case of the HR, necrotic features are often accompanied by the features of vacuolar cell death (see below).

Cytological hallmarks that distinguish plant necrosis from vacuolar cell death include mitochondrial swelling, the absence of the growing lytic vacuoles and an early rupture of the plasma membrane leading to shrinkage of the protoplast (Table 1; Figure 2).$^{9,19,32,33}$ Because there are no lytic vacuoles that clear the cytoplasm during necrosis, the corpses of necrotic cells remain largely unprocessed. A shrunken protoplast is one of the most easily detected features of plant necrotic cells (Figure 2). Time-course analysis of animal necrosis has revealed that the initial gain in cell volume (swelling) as a result of ion pump failure is followed by cell shrinkage. ${ }^{30}$ Plant cells have a cell wall that should counteract swelling of the protoplast at early stages of necrosis, which would therefore escape detection. ${ }^{32}$ However, an early loss of plasma membrane integrity can result in readily detectable protoplast shrinkage.

Necrosis is typically an acute cell death response that develops rapidly and takes from several minutes (toxic treatments) to up to a day, as seen in the HR. A recommended approach to diagnose plant necrosis is by combining EM analysis with the assessment of mitochondrial dysfunction (MMP and decreased levels of both oxygen consumption and ATP production) and both ROS and RNS accumulation (Table 2).

\section{Mixed and Atypical Modalities of Plant Cell Death}

HR with some features of vacuolar cell death. It has been long known that a programmed, localised cell death

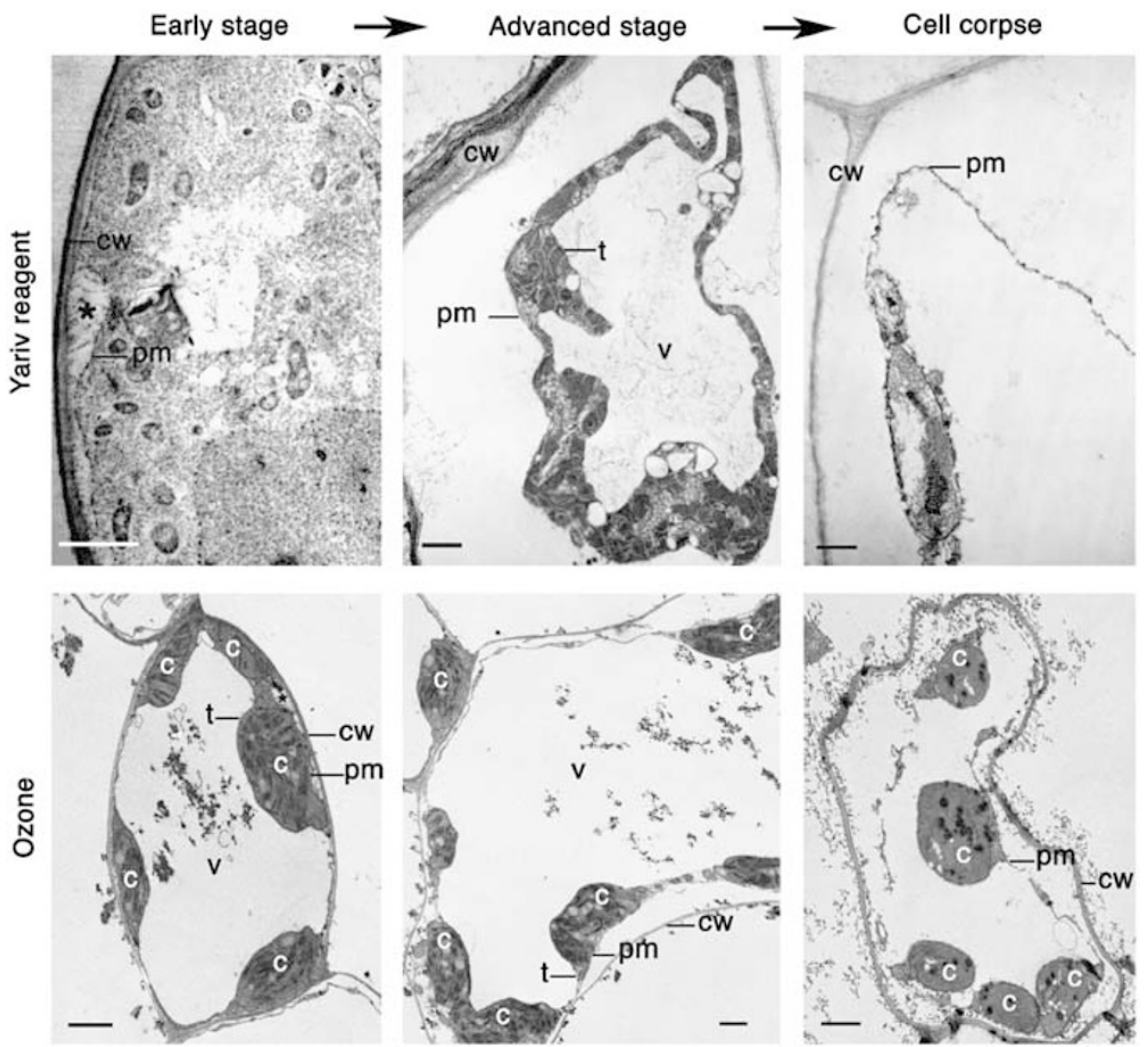

Figure 2 Necrotic cell death. Electron micrographs of Yariv reagent-induced death in the Arabidopsis cell culture ${ }^{53}$ (top panels) and ozone-induced death of the palisade cells in bean plants ${ }^{54}$ (bottom panels). Asterisks denote detachment of plasma membrane form the cell wall at early stage of cell death. c, chloroplast; cw, cell wall; pm, plasma membrane; t, tonoplast; v, vacuole. Scale bars, $2 \mu \mathrm{m}$. Pictures of Yariv reagent-induced cell death were kindly provided by Dr. Allan Showalter (Ohio University, Athens, $\mathrm{OH}, \mathrm{USA}$ ) and reproduced with permission from Gao and Showalter. ${ }^{53}$ Pictures of ozone-induced cell death were kindly provided by Dr. Franco Faoro (University of Milan, Milan, Italy) 
connected with the HR occurs at the site of successful recognition of biotrophic pathogens. Whether this cell death is the cause of restricted pathogen replication or a consequence thereof has been debated for decades. ${ }^{34}$ The nature of the HR cell death with respect to its morphology has also been debated. ${ }^{35-39}$ Most recently, HR cell death and pathogen replication restriction have been de-coupled by manipulation of metacaspase expression, showing that, for at least the pathogens tested, the elimination of the host cell death response does not lead to pathogen proliferation. ${ }^{40}$ HR cell death usually exhibits all characteristics of plant cell necrosis (Tables 1 and 2). However, HR cell death is at the same time often accompanied by the growth of lytic vacuoles and tonoplast rupture, which can require VPE from the vacuole in some cases. ${ }^{10,41}$ In addition, increased autophagic activity before HR cell death is apparently controlled by ATG genes, ${ }^{42}$ although the precise role of autophagy may differ depending on the particular HR cell death pathway being studied. ${ }^{43,44}$ Although autolytic components appear to be important for the HR cell death in some cases that have been studied, collapse of lytic vacuoles during the HR does not lead to complete clearance of the protoplast, as it does in vacuolar PCD. ${ }^{39,45}$

When discussing the relationship of the HR cell death to its correlated cytological features, and ultimately to the restriction of pathogen success, it is important to consider where the pathogens proliferate: for example, bacterial pathogens proliferate in the apoplast, outside the cell, while viruses proliferate within cells. Thus, vacuolar collapse can be effective to restrict viral pathogens, ${ }^{10}$ while discharge of defense proteins into the apoplast, accompanied by fusion of the tonoplast and plasma membrane, slows bacterial pathogens outside the cells. ${ }^{11}$

Shrunken protoplast and intact plasma membrane during victorin-induced cell death. A particular cell death, evoked by the fungal toxin victorin, is important because it has evolved to use the host HR as a means to kill cells, which are then 'digested' by the necrotrophic pathogen. Furthermore, similar to classic pathogen-induced HR, victorin sensitivity is dependent on an NB-LRR immune receptor. ${ }^{46}$ Although victorin-induced cell death in oat plants exhibits hallmarks of necrosis such as protoplast shrinkage and MMP, the shrunken protoplast is surrounded by an intact plasma membrane and the tonoplast retains its integrity. ${ }^{47}$ This suggests that initiation of the HR-related cell death can sometimes occur without the loss of membrane integrity. ${ }^{48}$

Mixture of vacuolar and necrotic hallmarks during self-incompatibility response. During self-incompatibility (SI) response in Papaver, an incompatible pollen tube is stopped by interactions with the pistil S-determinant that trigger a network of signalling events that converge to mediate PCD. ${ }^{49} \mathrm{SI}$ cell death exhibits some characteristics of vacuolar cell death, including alterations to the actin cytoskeleton, organelle engulfment and loss of vacuolar integrity. SI also has features of necrosis including swelling of the mitochondria and increase in $\left[\mathrm{Ca}^{2+}\right]_{\text {cyt. }}{ }^{49,50}$
A long time gap between cell death and corpse processing in cereal starchy endosperm. The cereal endosperm consists of the starchy endosperm surrounded by the aleurone cell layer. Cells of the starchy endosperm accumulate storage reserves and die during seed maturation, but their corpses remain unprocessed until germination. Upon seed germination, aleurone cells secrete hydrolytic enzymes that break down and mobilise the reserves accumulated in the dead starchy endosperm. ${ }^{51,52}$

\section{Recommendations to Authors, Reviewers and Editors of Scientific Journals}

Arbitrary and sometimes contradictory usage of terminology has been a problem in the field of plant cell death research. Here we have grouped together morphological characteristics that distinguish two major classes of cell death occurring in plants (Table 1). On the basis of this simplistic grouping, we suggest that terms 'vacuolar cell death' and 'necrotic cell death' (or 'necrosis') are used when referring to corresponding classes of cell death in plants. Because the HR cell death with autolytic features, victorin-induced cell death, and both starchy endosperm and SI cell death do not neatly fall into these two categories, we suggest that they are left as separate cell death modalities. The present classification is of course not static, but will be subjected to further revisions, especially when specific biochemical pathways and molecular identity of mediators for plant PCD are better defined.

We recommend that plant cell death researchers abandon terms such as 'apoptosis' or 'apoptotic-like'. We think such terminology is incorrect and misleading, because the features often cited are also found in other types of PCD, whereas the bona fide cytological characteristics of apoptosis (formation of apoptotic bodies and phagocytosis) are absent in plants.

Adequate choices of analytical methods are required to correctly diagnose a type of plant cell death (Table 2). Being a classic, cytological method, EM analysis provides excellent descriptive data on the changes in the dying cell for the initial classification of the particular morphotype. We encourage the use of EM in showing the temporal details of the cell death under study, such as the structure of organelles, formation of autophagosome-like structures, nuclear events and early detachment of plasma membrane from the cell wall (beginning of protoplast shrinkage).

We would furthermore advise that experiments with protoplasts are not used in isolation, but are supported by tests having a direct relationship to the physiologically relevant cell death in the model system.

Several of the recommendations formulated in the classification of animal cell death ${ }^{1}$ are also relevant to studies of plant cell death. For example, the term 'dead cell' should only be used for cells that are shown to be dead by specific staining, such as fluorescein diacetate or Evan's blue. We thus encourage the use of quantification of cell death along with the necessary statistical treatments to show significance for the reported data.

\section{Conclusion}

We recognise two major classes of cell death occurring in plant biology: (i) vacuolar cell death and (ii) necrotic cell death. 
Vacuolar cell death occurs during plant tissue and organ formation and elimination, although necrosis is typically found under abiotic stress, some forms of the HR-related cell death and cell death induced by necrotrophic pathogens. A few examples of cell death cannot be ascribed to either major class and therefore classified as separate modalities. This category includes HR cell death with autolytic features and victorin-induced cell death, as well as cell death occurring in starchy cereal endosperm and during SI response. Further studies using tools of genetics, biochemistry and cell biology are required to understand molecular mechanisms underlying variability of plant cell death morphology.

\section{Conflict of Interest}

The authors declare no conflict of interest.

1. Kroemer G, Galluzzi L, Vandenabeele P, Abrams J, Alnemri ES, Baehrecke EH et al Classification of cell death. Recommendations of the Nomenclature Committee on Cell Death 2009. Cell Death Differ 2009; 16: 3-11.

2. Kerr JF, Wyllie AH, Currie AR. Apoptosis: a basic biological phenomenon with wide-ranging implications in tissue kinetics. $\mathrm{Br} J$ Cancer 1972; 26: 239-257.

3. Clarke PGH. Developmental cell death: morphological diversity and multiple mechanisms. Anat Embryol 1990; 181: 195-206.

4. Leist M, Jäättelä M. Four deaths and a funeral: from caspases to alternative mechanisms. Nat Rev Mol Cell Biol 2001; 2: 589-598.

5. Lee $\mathrm{CY}$, Baehrecke EH. Steroid regulation of autophagic programmed cell death during development. Development 2001; 128: 1443-1455.

6. Hoyer-Hansen M, Bastholm L, Mathiasen IS, Elling F, Jäättelä M. Vitamin D analog EB1089 triggers dramatic lysosomal changes and Beclin 1-mediated autophagic cell death. Cell Death Differ 2005; 12: 1297-1309.

7. Fink SL, Cookson BT. Apoptosis, pyroptosis, and necrosis: mechanistic description of dead and dying eukaryotic cells. Infect Immun 2005; 73: 1907-1916.

8. Nunez R, Sancho-Martinez SM, Novoa JML, Lopez-Hernandez FJ. Apoptotic volume decrease as a geometric determinant for cell dismantling into apoptotic bodies. Cell Death Differ 2010; 17: 1665-1671.

9. Heath MC. Hypersensitive response-related death. Plant Mol Biol 2005; 44: 321-334.

10. Hatsugai N, Kuroyanagi M, Yamada K, Meshi T, Tsuda S, Kondo M et al. A plant vacuolar protease, VPE, mediates virus-induced hypersensitive cell death. Science 2004; 305: 855-858.

11. Hatsugai N, Iwasaki S, Tamura K, Kondo M, Fuji K, Ogasawara K et al. A novel membrane fusion-mediated plant immunity against bacterial pathogens. Genes Dev 2009; 23: 2496-2506.

12. Chichkova NV, Shaw J, Galiullina RA, Drury GE, Tuzhikov Al, Kim SH et al. Phytaspase, a relocalisable cell death promoting plant protease with caspase specificity. EMBO J 2010; 29: 1149-1161.

13. Marty F. Plant vacuoles. Plant Cell 1999; 11: 587-599.

14. Müntz K. Protein dynamics and proteolysis in plant vacuoles. J Exp Bot 2007; 58: 2391-2407.

15. Jones AM. Programmed cell death in development and defense. Plant Physiol 2001; 125 95-97.

16. Beers EP. Programmed cell death during plant growth and development. Cell Death Differ 1997; 4: 649-661.

17. Filonova LH, Bozhkov PV, Brukhin VB, Daniel G, Zhivotovsky B, von Arnold S. Two waves of programmed cell death occur during formation of development of somatic embryos in the gymnosperm, Norway spruce. J Cell Sci 2000; 113: 4399-4411.

18. Bozhkov PV, Filonova LH, Suarez MF. Programmed cell death in plant embryogenesis. Curr Top Dev Biol 2005; 67: 135-179.

19. van Doorn WG, Woltering EJ. Many ways to exit? Cell death categories in plants. Trends Plant Sci 2005; 10: 117-122.

20. Avci U, Petzold HE, Ismail IO, Beers EP, Haigler CH. Cysteine proteases XCP1 and XCP2 aid micro-autolysis within the intact central vacuole during xylogenesis in Arabidopsis roots. Plant J 2008; 56: 303-315.

21. Bethke PC, Lonsdale JE, Fath A, Jones RL. Hormonally regulated programmed cell death in barley aleurone cells. Plant Cell 1999; 11: 1033-1046.

22. Drew MC, He CJ, Morgan PW. Programmed cell death and aerenchyma formation in roots. Trends Plant Sci 2000; 5: 123-127.

23. Rubinstein B. Regulation of cell death in flower petals. Plant Mol Biol 2000; 44: 303-318.
24. Gunawardena AHLAN. Programmed cell death and tissue remodeling in plants. J Exp Bot 2008; 59: 445-451.

25. Fukuda H. Xylogenesis: initiation, progression, and cell death. Annu Rev Plant Physiol Plant Mol Biol 1996; 47: 299-345.

26. Courtois-Moreau CL, Pesquet E, Sjödin A, Muñiz L, Bollhöner B, Kaneda M et al. A unique program for cell death in xylem fibers of Populus stem. Plant J 2009; 58: 260-274.

27. Doelling JH, Walker JM, Friedman EM, Thompson AR, Vierstra RD. The APG8/12activating enzyme APG7 is required for proper nutrient recycling and senescence in Arabidopsis thaliana. J Biol Chem 2002; 277: 33105-33114.

28. Hanaoka H, Noda T, Shirano Y, Kato T, Hayashi H, Shibata D et al. Leaf senescence and starvation-induced chlorosis are accelerated by the disruption of an Arabidopsis autophagy gene. Plant Physiol 2002; 129: 1181-1193.

29. Kwon SI, Cho HJ, Jung JH, Yoshimoto K, Park OK. 2010. The RabGTPase RabG3b functions in autophagy and contributes to tracheary element differentiation in Arabidopsis. Plant J 2010; 64: 151-164.

30. Majno G, Joris I. Apoptosis, oncosis, and necrosis. An overview of cell death. Am J Pathol 1995: 146: 3-15.

31. Christofferson DE, Yuan J. Necroptosis as an alternative form of programmed cell death. Curr Opin Cell Biol 2010; 22: 263-268.

32. Jones AM. Does the plant mitochondrion integrate cellular stress and regulate programmed cell death? Trends Plant Sci 2000; 5: 225-230.

33. Scott I, Logan DC. Mitochondrial morphology transition is an early indicator of subsequent cell death in Arabidopsis. New Phytol 2008; 177: 90-101.

34. Kiraly Z, Barna B, Ersek T. Hypersensitivity as a consequence, not cause, of plant resistance to infection. Nature 1972; 239: 456-458.

35. Jones AM, Dangl JL. Logjam at the Styx: programmed cell death in plants. Trends Plant Sci 1996; 1: 114-119.

36. Greenberg JT. Programmed cell death in plant-pathogen interactions. Annu Rev Plant Physiol Plant Mol Biol 1997; 48: 525-545.

37. Richberg MH, Aviv DH, Dangl JL. Dead cells do tell tales. Curr Opin Plant Biol 1998; 1: 480-485.

38. Lam E, Kato N, Lawton M. Programmed cell death, mitochondria and the plant hypersensitive response. Nature 2001; 411: 848-853.

39. Mur LA, Kenton P, Lloyd AJ, Ougham H, Prats E. The hypersensitive response; the centenary is upon us but how much do we know? J Exp Bot 2008; 59: 501-520.

40. Coll NS, Vercammen D, Smidler A, Clover C, van Breusegem F, Dangl JL et al. Arabidopsis type I metacaspases control cell death. Science 2010; 330: 1393-1397.

41. Rojo E, Martin R, Carter C, Zouhar J, Pan S, Plotnikova J et al. VPE $\gamma$ exhibits a caspaselike activity that contributes to defense against pathogens. Curr Biol 2004; 14: 1897-1906.

42. Hofius D, Schultz-Larsen T, Joensen J, Tsitsigiannis DI, Petersen NHT, Mattson $\mathrm{O}$ et al. Autophagic components contribute to hypersensitive cell death in Arabidopsis. Cell 2009; 137: 773-783.193.

43. Liu Y, Schiff M, Czymmek K, Tallóczy Z, Levine B, Dinesh-Kumar SP. Autophagy regulates programmed cell death during the plant innate immune response. Cell 2005; 121: 567-577.

44. Yoshimoto K, Jikumaru $\mathrm{Y}$, Kamiya $\mathrm{Y}$, Kusano $\mathrm{M}$, Consonni $\mathrm{C}$, Panstruga $\mathrm{R}$ et al. Autophagy negatively regulates cell death by controlling NPR1-dependent salicylic acid signaling during senescence and the innate immune response in Arabidopsis. Plant Cell 2009; 21: 2914-2927.

45. Beers EP, McDowell JM. Regulation and execution of programmed cell death in response to pathogens, stress and developmental cues. Curr Opin Plant Biol 2001; 4: $561-567$.

46. Lorang J, Cuesta-Marcos A, Hayes PM, Wolpert TJ. Identification and mapping of adultonset sensitivity to victorin in barley. Mol Breeding 2010; 26: 545-550.

47. Curtis MJ, Wolpert TJ. The victorin induced mitochondrial permeability transition precedes cell shrinkage and biochemical markers of cell death and shrinkage occurs without loss of membrane integrity. Plant J 2004; 38: 244-259.

48. Krzymowska M, Konopka-Postupolska D, Sobczak M, Macioszek V, Ellis BE, Hennig J. Infection of tobacco with different Pseudomonas syringae pathovars leads to distinct morphotypes of programmed cell death. Plant $J$ 2007; 50: 253-264.

49. Bosch M, Franklin-Tong VE. Self-incompatibility in Papaver: signalling to trigger PCD in incompatible pollen. J Exp Bot 2008; 59: 481-490.

50. Geitmann A, Franklin-Tong VE, Emons AC. The self-incompatibility response in Papaver rhoeas pollen causes early and striking alterations to organelles. Cell Death Differ 2004; 11: 812-822.

51. Fath A, Bethke P, Lonsdale J, Meza-Romero R, Jones R. Programmed cell death in cereal aleurone. Plant Mol Biol 2000; 44: 255-266.

52. Young TE, Gallie DR. Programmed cell death during endosperm development. Plant Mol Biol 2000; 44: 283-301.

53. Gao M, Showalter AM. Yariv reagent induces programmed cell death in Arabidopsis cell cultures and implicates arabinogalactan protein involvement. Plant $J$ 1999; 19: 321-331.

54. Faoro F, Iriti M. Plant cell death and cellular alterations induced by ozone: key studies in Mediterranean conditions. Environ Pollut 2009; 157: 1470-1477. 